\section{Antibiotic Sensitivity Testing}

It is notorious that tests of bacterial sensitivity to antibiotics are done in an extraordinary variety of ways. Many variations of the usual methods are misconceived and inadequately controlled, or lend themselves to misinterpretation. False information has thus often been provided to clinicians, of which they have sometimes not been slow to complain. There are indeed some who have expressed the view that laboratory reports on this subject are better disregarded.

This problem was considered by an expert committee of the World Health Organization which met in 1960, and its report $^{1}$ recommended the definition of a standard routine method employing paper discs. The factors requiring control were defined, and the main features of a suitable method were laid down in some detail, but many questions on such matters as choice of antibiotics, disc contents, culture media, and procedures for interpretation and reporting were necessarily left unsettled. The task of furthering this project was taken up almost immediately by a working group sponsored by W.H.O., which has owed much to the leadership of Dr. Hans Ericsson, of Stockholm. From this an international collaborative study developed, in which representatives of many countries have taken part at various times. This study enabled almost every aspect of the subject to be investigated thoroughly, and the results of actual tests performed by prescribed methods in many different laboratories have been compared. The working group has had two formal meetings in Geneva and two in Stockholm, and informal meetings of such members of the group as were present during international conferences in Naples, Montreal, Vienna, Paris, and elsewhere. Various findings by individual workers have been published, but the progress of the study as a whole has been recorded only in reports for private circulation, though many bacteriologists have been aware of some of their contents.

This situation has now been remedied. A full report of the entire enterprise, with recommendations to which it leads, has been published by H.M. Ericsson and J. C. Sherris. ${ }^{2}$ Techniques are proposed for two dilution methods, and for a disc method which can be used either as a routine or to check the accuracy of another already in use. This employs large plates and high-content discs, and thus resembles the KirbyBauer method, ${ }^{3}$ in which diameters of inhibition zones are translated into categories of sensitivity or resistance by reference to tables. The present report proposes that these diameters should be converted to minimum inhibitory concentrations and thus into such categories, but no actual instructions are given about how this is to be done.

One of the tasks of the working group was to construct regression lines (plots of zone diameters against minimum inhibitory concentrations determined by a dilution method) for 12 antibiotics, and these enable the conversion to be made. These lines are reproduced, but not all are considered satisfactory, and among various proposals for further work is that there should be complete and elaborate restudy of this aspect of the subject. It is also suggested that there should be national reference laboratories with the duty of "preparing complete descriptions of current recommended methods and interpretative schemes", and of supplying fully characterized reference cultures with which the performance of a method can be checked. The proposal that each country should now shoulder this burden independently is in conflict with the policy of the original W.H.O. Committee, whose intention was that reliable methods should be proposed for "universal adoption". Nevertheless, it seems that steps have been or are being taken to carry out this proposal in France, Germany, Sweden, and the United States.

The slow progress made may be attributed to another departure from the original intention at Geneva. This was understood to be that a relatively simple procedure for a disc test should be so defined as to eliminate the known common sources of serious error. That could have been done in far less time, and the adoption of such a method might already have enabled innumerable illnesses to be shortened and even have helped to save many lives. The procedure which has been studied is more elaborate, much more costly in materials, requires the most rigid standardization, particularly in regard to the composition of the culture medium (another problem not yet finally settled), and its interpretation is still to some extent the subject of controversy. The kind of laboratory where most of the mistakes are made will have difficulty in practising it successfully. But this is a serious proposal, based on very extensive study and backed by leading world authorities, and at present there is no alternative to it. Since one thing on which almost everyone is agreed is that something needs to be done, Great Britain cannot remain idle while other countries implement the W.H.O. proposals. This is surely a matter in which official action is required to determine British policy.

\footnotetext{
Standardization of Methods for Conducting Microbic Sensitivity Tests. W. H.O. Technical Report Series, No. 210, 1961 .

Ericsson, H. M., and Sherris, J. C., Acta Pathologica et Microbiologica Scandinavica, 1971, Supplement No. 217.

3 Bauer, A. W., Kirby, W. M. M., Sherris, J. C., and Turck, M., American Fournal of Clinical Pathology, 1966, 45, 493.
}

\section{Hepatic Hypoglycaemia}

Among patients with fulminant hepatic failure due to viral hepatitis or the toxic effects of drugs occasional cases appear with spontaneous and profound hypoglycaemia. Since frequent monitoring of the blood sugar is still not a routine practice in the management of these patients, hypoglycaemia may pass unrecognized, its symptoms being attributed to hepatic encephalopathy. ${ }^{1}$ This is tragic, for the hypoglycaemia leads to further brain damage. Restoration of the blood sugar to normal can result in an improvement in the level of consciousness and in the signs of disturbed function of the pyramidal tracts.

The cause of this hypoglycaemia is uncertain. Insulin is secreted into the portal system and probably much of it is enzymatically destroyed by the liver. In a case of fulminant hepatitis reported by $\mathbf{R}$. L. Sampson and colleagues, ${ }^{2}$ of Cape Town, enormous amounts of sugar $(2.5 \mathrm{~kg})$ were given at one stage over 24 hours with persistence of the hypoglycaemia. This together with the finding of a high level of insulin in the plasma suggested to them that failure of degradation of insulin by the liver played an important part in the hypoglycaemia. The patient subsequently died, and at necropsy the pancreatic islets appeared larger than normal, with increased cellular cytoplasm and nuclear variation, raising the possibility of enhanced production of insulin. Other mechanisms have been suggested. Synthesis of glycogen or its storage in the liver may have failed, as may its subsequent breakdown, which is the main process responsible for maintaining the blood sugar in the fasting state.

A recent report of P. Felig and colleagues ${ }^{3}$ at Yale is of considerable interest, for they showed that hypoglycaemia 
can also occur in acute viral hepatitis uncomplicated by massive or subacute hepatic necrosis. In the 15 consecutive patients studied the mean plasma glucose was significantly lower than in the control group, and in eight patients the plasma glucose fell below $60 \mathrm{mg} / 100 \mathrm{ml}$. None showed the classical features of hypoglycaemia such as palpitation, hunger, syncope, or sweating, but a few complained of general weakness and headache. The occurrence of hypoglycaemia in several patients with a normal prothrombin time and serum albumin level indicates that the glucose-regulating role of the liver cell may be compromised while its other functions in protein synthesis remain unimpaired. The hypoglycaemia could not be attributed to hyperinsulinism consequent on inadequate hepatic degradation of insulin. Indeed, the levels of plasma insulin were decreased by $30 \%$ in the eight hypoglycaemic patients, and the direct linear correlation found in the whole group between the fasting blood sugar and insulin levels indicated that the concentration of insulin in the blood was appropriately reduced in the patients in whom the hypoglycaemia developed. A reduction of glycogen in the liver was observed on biopsy. The decrease was due both to loss of parenchymal cells and to decreased content of glycogen in the remaining cells. In normal persons the plasma glucose rises by at least a third between 15 and 45 minutes after administration of glucagon, but in the patients with hepatitis the rise of plasma glucose was considerably less. This is consistent with impairment of glycogen synthesis in the liver in these patients.

The administration of glucagon to normal persons also produces a prompt reduction in plasma amino-acids. The decrease is greatest for alanine and glycine, and this is considered on other evidence to be largely a reflection of enhanced uptake of amino-acids in the liver and their incorporation with glucose. However, in their patients with hepatitis Felig and colleagues found no consistent reduction in the concentration of amino-acid after giving glucagon. This finding underlines the importance of the liver in determining the level of amino-acid in response to glucagon, and it also suggests that, in patients with viral hepatitis, gluconeogenesis as well as glycogen synthesis is severely impaired. These results need to be confirmed, for other workers in an earlier study ${ }^{4}$ found a diabetic type of glucose tolerance curve in 9 out of 10 patients with hepatitis. It returned to normal in seven of these patients when they were re-examined after recovering from the jaundice.

Hypoglycaemia occurs in other liver conditions, including primary carcinoma of the liver. The mechanism suggested to account for it is diversion of glucose to the tumour. ${ }^{5}$ In one study a decreased output of glucose from the liver was found in patients with primary hepatic carcinoma. 6 Such tumours may produce an insulin-like substance or one that blocks the release of glucose by the liver. There is increasing evidence that primary liver tumours can synthesize metabolically active hormones or homone-like substances, as do tumours arising in other organs. Increased levels of chorionic gonadotrophin have been found in the serum of children with hepatoblastoma and precocious puberty. In a recently reported adult patient with hypercalcaemia and a primary hepatoma direct evidence that the tumour was actively secreting a parathyroid-hormone-like polypeptide into the circulation was obtained during operation.?

Hypoglycaemia also occurs after ingestion of large quantities of alchohol and can cause coma and death. Again the mechanism is uncertain, but increased utilization of glycogen stores during the metabolism of alcohol in a poorly nourished person and decreased gluconeogenesis have been suggested. ${ }^{2}$ Hypoglycaemia can occur without other clinical signs of acute liver damage. One liver condition in which the mechanism is known is glycogen storage disease type 1 (von Gierke's disease). Glycogen breakdown is impaired by a deficiency in glucose-6-phosphatase.

In contrast, the changes associated with cirrhosis of the liver are in the opposite direction, with hyper- rather than hypoglycaemia, and these patients are liable to diabetes. 89 A variety of explanations for this have been suggested, and they have recently been reviewed by J. R. Collins and colleagues. ${ }^{10}$ In their own patients they found inappropriately high serum levels of insulin in response to a glucose load, with a diminished glucose response to injected insulin. By contrast the plasma concentrations of amino-acids and free fatty acids showed a normal response to injected insulin. These observations suggested that there might be resistance to the regulatory action of insulin on glucose metabolism. Further study of the changes in acute and chronic liver diseases should help to solve some of the problems of the regulation of glucose homoeostasis.

Williams, R., British Medical fournal, 1971, 1, 213.

Samson, R. I., Trey, C., Timme, A. H., and Saunders, S. J., Gast:oenterology, 1967, 53, 291.

3 Felig, P., Brown, W. V., Levine, R. A., and Klatskin, G., New England fournal of Medicine, 1970, 283, 1436.

Berkowitz, D., Blinkoff, B., and Glassman, S., Gastroenterology, 1966,

$50,830$.
5 Madzean, A. J. S., and Yeung, T. T., Archives of Internal Medicine, $1956,98,720$.

6 Laundau, B. R., Wills, N., Craig, J. W., Leonards, J. R., and Moriwaki, T., Cancer (Philadelphia), 1962, 15, 1188.

Knill-Jones, R. P., Buckle, R. M., Parsons, V., Calne, R. Y., and Williams, R., New England Fournal of Medicine, 1970, 282, 704.

8 Creutzfelt, W., in Progress in liver diseases, Vol. 3, ed. H. Popper and F. Schaffner. London, Heinemann Medical, 1970

9 Megyesi, C., Samols, E., and Marks, V., Lancet, 1967, 2, 1051.

10 Collins, J. R., Lacy, W. W., Stiel, J. N., and Crofford, O. B., Archives of Internal Medicine, 1970, 126, 608.

\section{Community Medicine}

Medicine provides for the needs of both the individual and the community, and it cannot fully realize its mission unless it has regard to both. In every illness there are social and other factors which must be taken into account if the maximum benefit from medical care is to be obtained. During the past 125 years the public health services have been largely preoccupied with the control of infection, raising the environmental standards of living, and providing medical services to meet particular needs. The record of achievements shows that even with their often limited resources the British public health services have done a good job, for which much of the credit must go to the dedicated medical men and women who directed and served in them. Now with the changing patterns of disease and medical care the role of the community physician has emerged and is being widely and enthusiastically welcomed if not yet altogether clearly defined.

The Royal Commission on Medical Education ${ }^{1}$ attempted, with obvious difficulty, to define "community medicine," but it was in no doubt about the essential part that community medicine has to play in the provision of medical care and in medical teaching. The need for a community physician with a detailed knowledge of local health problems and involved with his clinical colleagues in planning and developing health and other services to meet these needs has been emphasized by two chief medical officers. ${ }^{2-4}$ The importance of the community physician as the link between the hospital services, the general practitioners, and 